\title{
Immunohistochemical Characterization of Monoclonal Antibodies (PDs) as Markers of the Periderm in the Developing Chicken Embryo
}

\author{
Keitaro Isokawa ${ }^{1,2}$, Junichi Honma ${ }^{2}$, Keita Murakami², Mizuho Tanaka², Hidehiro Aoki², \\ Hideyuki Takahashi ${ }^{2}$, Hirokazu Yokoyama ${ }^{2}$, Seiichi Endo ${ }^{2}$ and Yoshihisa Toda ${ }^{1,2}$
}

(Received 2 July and accepted 27 September 1996)

Key words: development, ectoderm, epidermis, periderm, monoclonal antibody, desmosome

\begin{abstract}
The outermost surface cell layer of the developing embryo, the periderm, arises from the initial single layer of ectoderm and is eventually exfoliated from the stratified epidermis, which has the same ectodermal origin. In this study, monoclonal antibodies against chicken limb bud ectoderm were generated and screened for those which stained the periderm. Four separate antibodies termed PD2, 3, 7 and 9 were obtained from 180 mixed hybridomas. These PD antibodies stained the periderm selectively at all stages examined (stage 20-42). By correlating the results of immunohistochemistry with observations made by transmission electron microscopy, it was revealed that PD antibodies stained both the squamous periderm at an early stage and rounded bulging peridermal cells just before exfoliation. Therefore we feel that PD antibodies may be useful in further systematic investigations of the development and function of the chicken embryonic periderm.
\end{abstract}

\section{Introduction}

The outer surface of avian and mammalian embryos consists of a single basal cell layer covered by a specialized epithelium known as the periderm, which arises from a simple epithelium-like ectoderm in the early stages of development ${ }^{[1]}$. It is reported that the periderm is not discernible until 7 days post coitum in the cat embryo ${ }^{[2]}$ and until 4 weeks of gestation in the human embryo ${ }^{[3]}$.

During subsequent development, the initial embryonic epidermis consisting of peridermal cells and incompletely keratinized basal cells differentiates into the basal, intermediate (or suprabasal) and peridermal cell layers; this is followed by a progressive increase in the number of keratinocyte layers, until finally the distinct basal, spinous, granular and cornified layers characteristic of the mature epidermis are formed ${ }^{[4,5]}$. In the meantime, the periderm is sloughed from the surface of the embryo or fetus. This shedding of the periderm occurs around 18 days of gestation in mice and 23-24 weeks in humans ${ }^{[6-8]}$. Periderm exfoliation seems to be genetically regulated because it is sloughed in a temporally relevant manner from fetal epidermis maintained in an organ culture system ${ }^{[9]}$.

The function of the periderm remains speculative. One of the most traditional assumptions is that it may have an osmoregulatory function to control water permeability and support the transition from an aqueous to a gaseous environment at birth (or hatching) ${ }^{[10]}$. Recently, active involvement of the periderm in glucose uptake from the amniotic fluid ${ }^{[1]}$ and in morphogenesis ${ }^{[12]}$, especially limb formation ${ }^{[13]}$, has been emphasized, but none of these interpretations is yet conclusive.

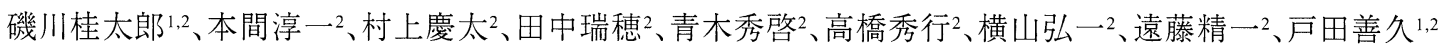

1 Division of Electron Microscopy, Dental Research Center, Nihon University

2 Department of Anatomy, Nihon University School of Dentistry

To whom all correspondence should be addressed: Dr. Keitaro Isokawa

Division of Electron Microscopy, Dental Research Center, Nihon University 1-8-13 Kanda-Surugadai, Chiyoda-ku, Tokyo 101, JAPAN 
In the present study, we generated monoclonal antibodies selective to the periderm of chicken embryo and characterized them immunohistochemically in order to assess their possible use for systematic investigations on the development and function of the periderm.

\section{Production and screening of monoclonal antibodies}

\section{Materials and Methods}

Fertilized white Leghorn eggs (Ohata Hatchery, Shizuoka) were incubated and staged according to Hamburger and Hamilton ${ }^{[14]}$. Limb ectoderm from stage 20-22 embryos was collected and sonicated in $200 \mu \mathrm{l}$ of sterile saline with an equal volume of Freund's complete adjuvant (Gibco BRL). Immunization to a female BALB/c mouse (6 weeks old, approximately $16-19 \mathrm{~g}$ in weight) was done intraperitoneally three times with an interval of 3 weeks. Freund's incomplete adjuvant (Gibco BRL) was used for booster immunogen preparations. Three days after the last injection, the mouse was killed and the spleen cells were dissociated and fused with mouse myeloma cells (P3X63Ag8U.1) according to the standard technique ${ }^{[15]}$. Screening of antibody activity in undiluted supernatants of the growing hybridomas was carried out by indirect immunofluorescence on unfixed fresh frozen limb bud sections from stage 20-22 chicken embryos. Selected hybridomas were cloned by limiting dilution in 96-well culture plates using peritoneal macrophage-conditioned medium as a growth promoter.

\section{Determination of antibody class}

Hybridoma-conditioned media, which were shown to be positive for the periderm after single-cell cloning, were used for determination of the immunoglobulin subtype. For this purpose, heavy chain-specific rabbit anti-mouse IgG1, IgG2a, IgG2b, IgG3, IgM or IgA were diluted to 1 $\mu \mathrm{g} / \mathrm{ml}$ with $50 \mathrm{mM}$ sodium carbonated buffer $(\mathrm{pH} 9.6$ ), pipetted into a microtiter plate (Falcon 3915; Becton Dickinson) and incubated for $1 \mathrm{~h}$ at $37^{\circ} \mathrm{C}$. Non-specific binding sites were blocked with $1 \%$ bovine serum albumin (BSA; Sigma) in $20 \mathrm{mM}$ Tris $-500 \mathrm{mM} \mathrm{NaCl}-0.05 \%$ Tween $20^{\mathrm{TM}}$ (TBSS-T, $\mathrm{pH}$ 7.4) for $4 \mathrm{~h}$ at room temperature. Conditioned media to be tested were pipetted at $100 \mu \mathrm{l}$ per well and incubated for $1 \mathrm{~h}$. After extensive washing with TBSS-T, alkaline phosphateconjugated goat anti-mouse IgG (whole molecule, Cappel) diluted 1:600 with TBSS-T was pipetted and incubated for $30 \mathrm{~min}$. A solution of $p$-nitrophenyl phosphate (Sigma) at $1 \mathrm{mg} / \mathrm{ml}$ in $10 \mathrm{mM}$ diethanolamine buffer ( $\mathrm{pH} 9.6$ ) containing $0.5 \mathrm{mM} \mathrm{MgCl} 2$ was added, and the absorbance at $405 \mathrm{~nm}$ was determined using a microplate reader (Model 450; Bio-Rad).

\section{Immunoreagents}

Monoclonal antibodies which stained the periderm were designated PD2, PD3, PD7 and PD9. An antibody (termed $\mathrm{BM}$ ) reactive with the basement membrane was also used for comparison. Undiluted hybridoma-conditioned media for these antibodies were used for immunohistochemistry. Mouse anti-desmoplakin-1 (DP2.17, IgG1; Progen) was purchased and used at 1:10 dilution. For immunohistochemical detection, fluorescent isothiocyanate (FITC)conjugated goat anti-mouse IgM ( $\mu$ chain-specific; Cappel) and FITC-conjugated goat anti-mouse IgG (whole molecule; Cappel) were diluted 1:100 with 1\% BSA in phosphate-buffered saline (PBS, $\mathrm{pH}$ 7.4) and used as secondary antibodies.

\section{Immunostaining protocol}

Unfixed chicken embryos at stages from 20 to 42 were cryoprotected with a graded series of sucrose $(10 \%, 15 \%$ and $20 \%)$ in PBS, embedded in Tissue-Tek ${ }^{\mathrm{TM}}$ OCT compound (Miles) and frozen in liquid nitrogen-cooled 2-methyl butane (Wako). Cryosections, $8 \mu \mathrm{m}$ thick, were prepared with a cryostat (Cryocut 1800; Reihert-Jung). After blocking treatment with $1 \%$ BSA in PBS for $1 \mathrm{~h}$, the sections were incubated in undiluted hybridoma-conditioned media for $1 \mathrm{~h}$. 
Binding of the primary antibody was visualized by incubating sections with FITC-conjugated secondary antibodies for $1 \mathrm{~h}$. The sections mounted in $90 \%$ glycerol in PBS containing $0.2 \mathrm{M} n$ propylgallate $^{[16]}$ were examined with a Nikon Optiphot ${ }^{\mathrm{TM}}$ epifluorescence microscope. For controls, the primary antibody was omitted or replaced with non-immune mouse IgG.

\section{Electron microscopy}

Chicken embryos at stages 24,39 and 42 were fixed with $2.5 \%$ glutaraldehyde in PBS (pH 7.3) for $2 \mathrm{~h}$ at $4{ }^{\circ} \mathrm{C}$ and rinsed. Specimens were post-fixed in $1 \% \mathrm{OsO}_{4}$ in $0.1 \mathrm{M}$ phosphate buffer $(\mathrm{pH} 7.3)$ for $2 \mathrm{~h}$ at room temperature, routinely dehydrated in graded alcohols and propylene oxide, and embedded in Spurr's low-viscosity resin ${ }^{[17]}$. Thin sections (75 $\mu \mathrm{m}$ in thickness) were cut along the proximo-distal axis of the developing leg bud using a Sorvall MT-5000

ultramicrotome. Sections were contrasted with uranyl acetate and lead double-staining and examined in a Hitachi $\mathrm{H}-800$ electron microscope at an accelerating voltage of $75 \mathrm{kV}$.

\section{Results}

The fusion resulted in 180 wells that contained successful hybrids. The media from 65 wells stained chicken tissue immunohistochemically, but only 4 out of these 65 were selective for periderm, which was the focus of the present study. These 4 mixed hybridomas were cloned separately by limiting dilution, resulting in the same numbers of single-cell clones which were designated PD2, PD3, PD7 and PD9, respectively. The immunoglobulin class of these PD antibodies was exclusively IgM.

The staining with all four PD antibodies was selective for most, but not all epithelia. In the limb bud ectoderm, intense staining was observed in the surface cell layer, the periderm. However, punctate weak staining was also observed in the basal portion of the ectoderm. Desmoplakin-1 staining representing desmosome localization was observed mostly in the apical portion of the ectoderm, probably at the junctions between peridermal cells. The embryonic mesenchyme was completely unreacitve with PD antibodies. These observations were made from stage 20, the earliest stage examined, until stage 26 (Fig. 1). It appeared that PD3 and PD7 had higher affinity for the antigen molecule(s) than PD2 and PD9, because the staining intensity, especially that for the basal particulates, was greater in PD3 and PD7 immunohistochemistry. This assumption, however, needs to be examined quantitatively using purified preparations of these antibodies.

At stage 39 (approximately 13 days of development), the periderm was stained intensely by PD antibodies, but the basal and probably intermediate cells in the differentiating embryonic epidermis were only weakly stained. Mesenchymal tissues were completely negative (Fig. 2A). Accumulation of desmosomes in the periderm became marked (Fig. 2B). At stage 42 (approximately 17 days of development), the periderm remained intensely stained. The layers of keratinocytes which were not observed clearly in the fluorescent images were only weakly stained (Fig. 2C). Desmoplakin-1 staining was intense in the periderm and moderate in the underlying keratinocyte layers (Fig. 2D).

Monoclonal PD antibodies stained not only limb bud periderm but also the periderm covering other parts of the embryo such as the head, branchial arches, dorsal trunk and tail bud. Thus, most of the chicken embryo periderm was detectable with PD antibodies, except for the periderm covering the apical ectodermal ridge (AER) of the developing limb bud, which was negative. It appeared that negative staining of the AER periderm by PD antibodies correlated with the functional activity of AER, since the periderm covering the AER showed restoration of positive staining after stage 26, when the AER became less distinct morphologically (Fig. 3).

Along with the periderm, the gut endoderm and coelomic epithelial cells were also stained by PD antibodies. At more advanced stages, the outermost cell layer of feather follicles facing the dermal pulp or surrounding mesenchyme was intensely stained (Fig. 3). 

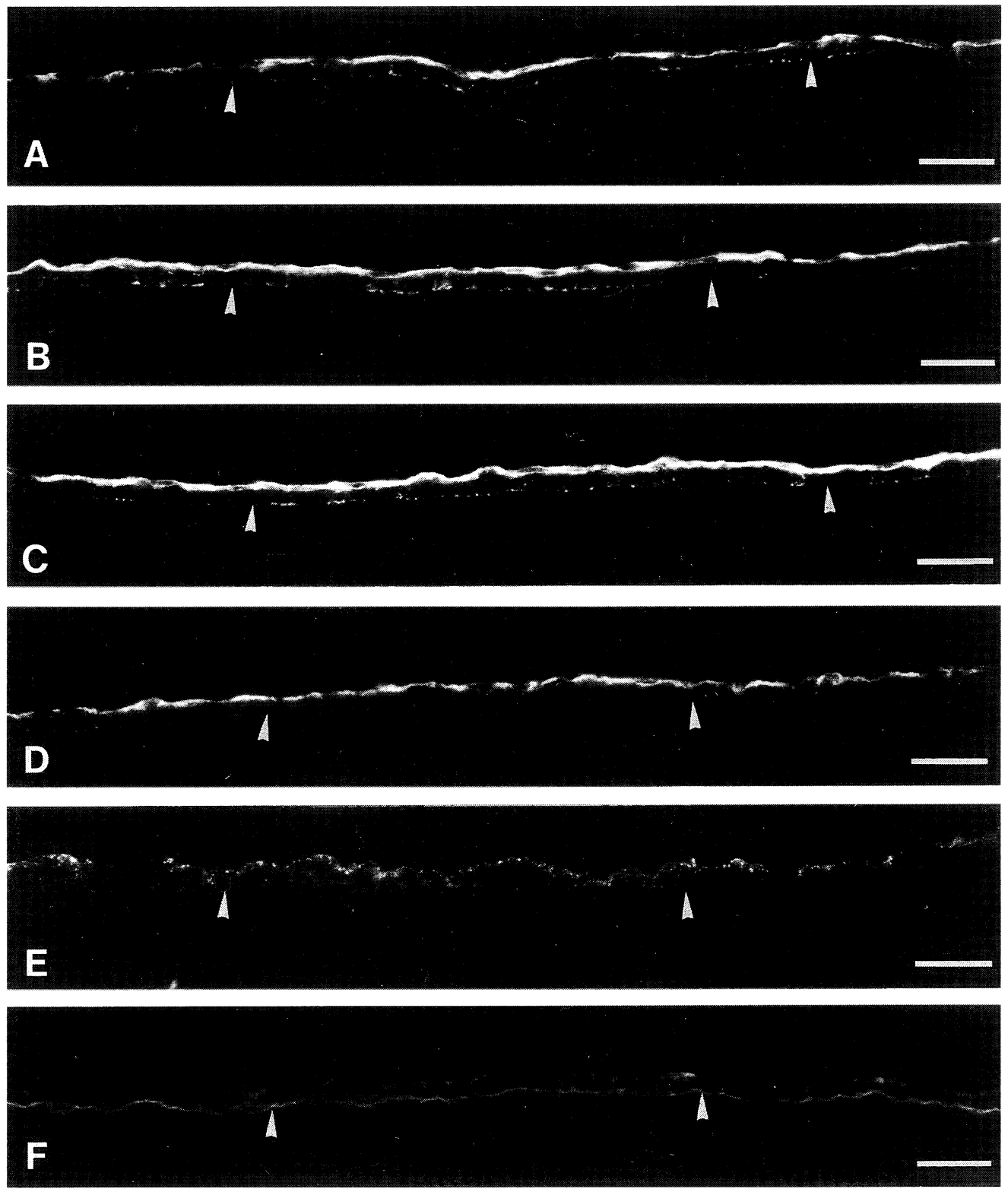

Fig. 1 Dorsal ectoderm of a stage 26 leg bud stained with PD2 (A), PD3 (B), PD7 (C), PD9 (D), anti-desmoplakin-1 (E) and BM (F). Arrowheads indicate the ecto-mesenchymal junction. BM antibody stains the basement membrane in the junction. Note that intense staining by PD antibodies is selective for the periderm (A-D). Bars represent $200 \mu \mathrm{m}$. 

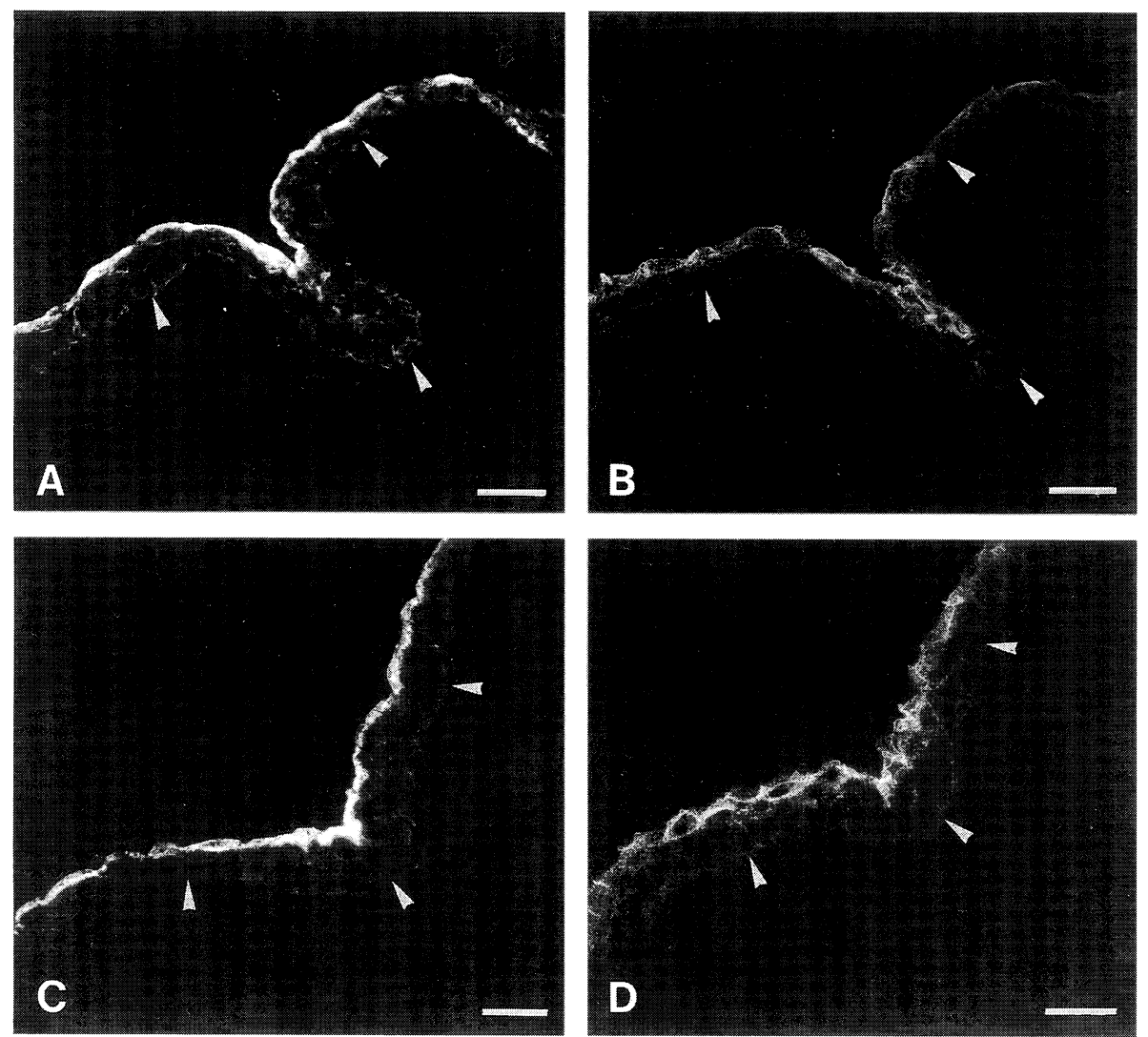

Fig. 2 Dorsal ectoderm of stage 39 (A, B) and 42 (C, D) leg buds stained with PD7 (A, C) and anti-desmoplakin-1 (B, D). Arrowheads indicate the ecto-mesenchymal junction. Bars represent $200 \mu \mathrm{m}$.

The embryonic epidermis in the developing limb bud was examined ultrastructually. That of the youngest embryos examined (stage 20) had already differentiated beyond the single-layered epithelium and consisted of basal and intermediate cell layers and the surface periderm. The peridermal cells were conjugated to each other and to the underlying epidermal cells by junctions of the desmosome type. The electron density of the periderm was frequently higher than that of the underlying future keratinocytes. This may be accounted for by the organization of microfilaments, which is reported to shape the flattened peridermal cells ${ }^{[3]}$. The histological architecture of the epidermis did not change markedly until stage 39 (Figs. 4A and B), but its compactness increased gradually. In other words, the wide intercellular spaces usually found within the epidermis at earlier stages became less pronounced in later development. By stage 42, stratification of keratinocytes had progressed and the surface keratinocyte layer which was just beneath the periderm showed ultrastructually apparent cornification. The peridermal cells became rounded bulging cells containing abundant glycogen, which was compartmentalized within the cytoplasmic spaces. The surface of the periderm was uniformly covered with many cytoplasmic protrusions or microvilli (Fig. 4C). 

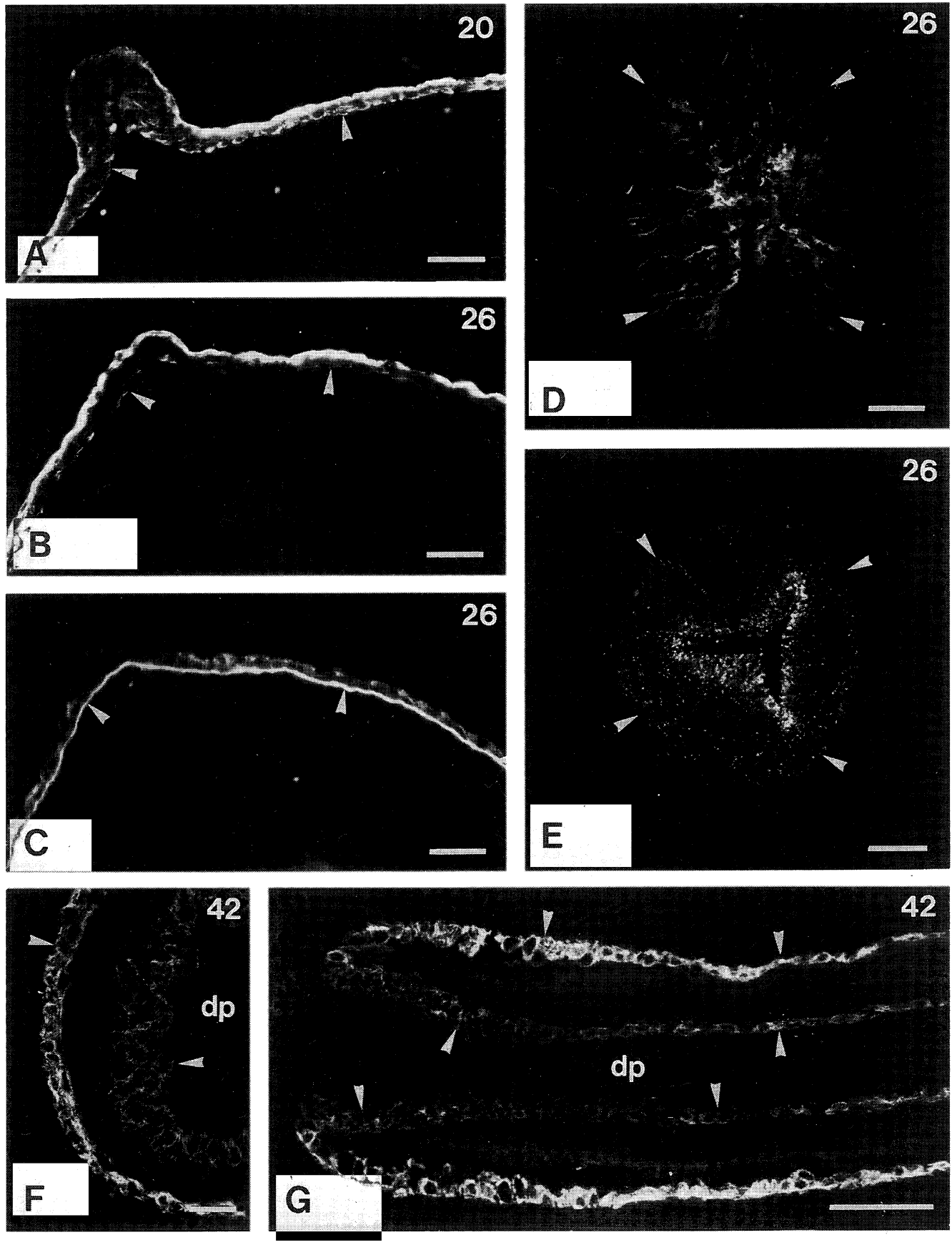

Fig. 3 Apical ectodermal ridge (A, B, C), gut epithelium (D, E) and feather follicle $(F, G)$ stained with PD3 (D, F), PD7 (A, B, G), BM (C) and anti-desmoplakin-1 (E). Stages of specimens are labeled on the upper right. A feather follicle is shown in cross- $(F)$ and longitudinal $(G)$ sections. Arrowheads indicate the ecto-mesenchymal junction. BM antibody stains the basement membrane in the junction. dp, dermal pulp of feather follicle. Bars represent $200 \mu \mathrm{m}$. 

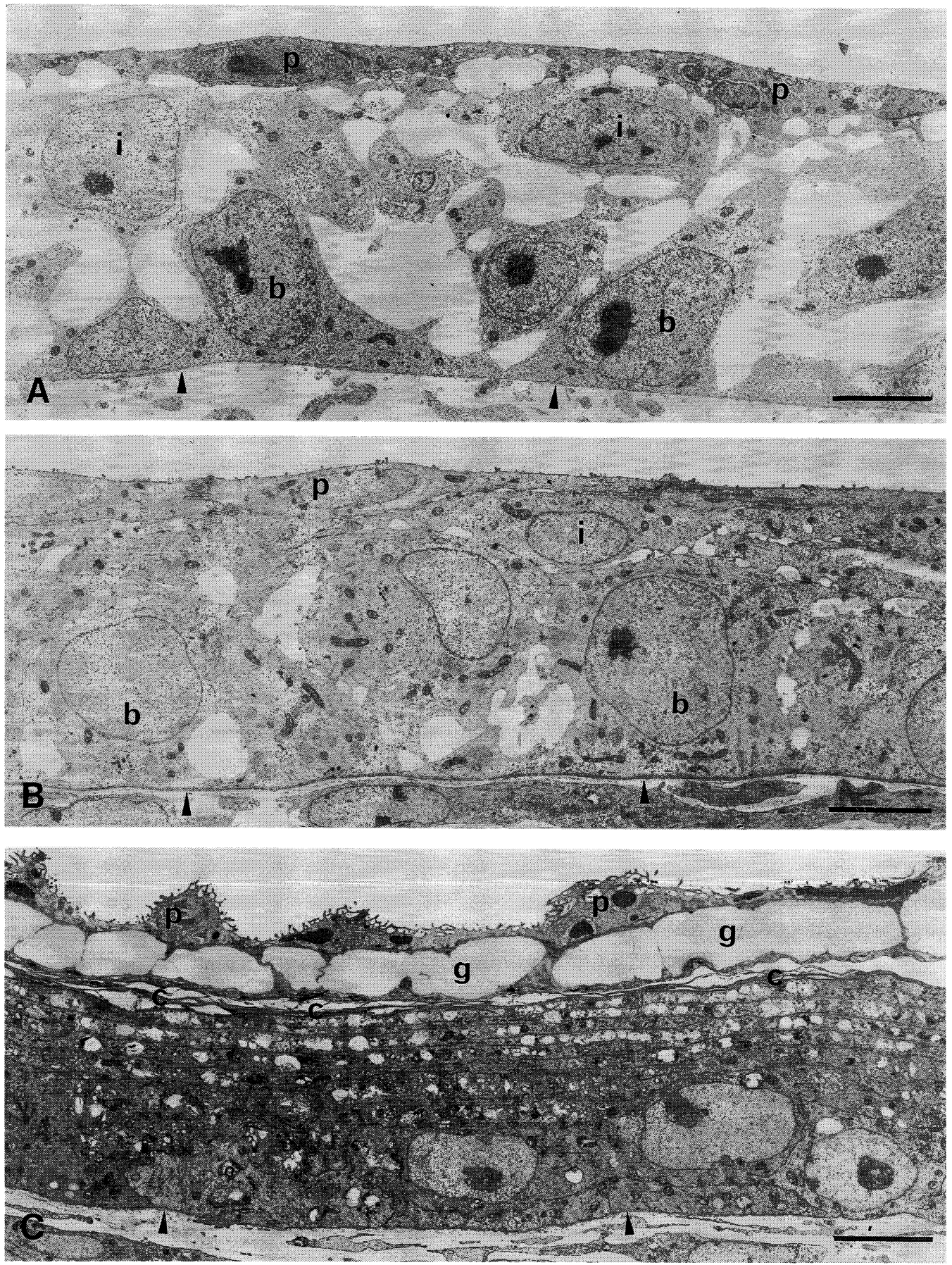

Fig. 4 Ultrastructure of embryonic non-ridge epidermis in the developing limb bud at stages 24 (A), 39 (B) and 42 (C). In A and B, the periderm (p) and the intermediate (i) and basal (b) cells are shown. In $\mathrm{C}$, the periderm $(\mathrm{p})$ with microvilli and a large amount of glycogen $(\mathrm{g})$ is about to be sloughed from the surface cornified layer (c) of keratinocytes. Arrowheads indicate the ecto-mesenchymal junction. Bars represent $5 \mu \mathrm{m}$. 


\section{Discussion}

The monoclonal antibodies generated in the present study stained the periderm intensely in a sufficiently selective manner. Weak punctate staining detected in the intermediate and basal cell layers as well as the stratified keratinizing cell layers did not hinder identification of the periderm in a practical setting. By correlating the immunohistochemical results (Fig. 2) with the ultrastructural findings (Fig. 4), it was revealed that the PD antibodies stained not only squamous peridermal cells in the early stages but also the rounded bulging periderm possessing many cytoplasmic protrusions. Such non-squamous periderm containing abundant glycogen at stage 42 was considered to be sloughed off soon, because the underlying layers of keratinocytes showed apparent cornification which appeared to promote exfoliation by loosening the junction between the periderm and the keratinized cells. Therefore, PD antibodies are useful as marker probes for systematic investigations of periderm histogenesis.

Moreover, the functional heterogeneity of the periderm might be demonstrable using PD antibodies. In the present study, the periderm of the AER was positive at least until stage 24 but negative at stage 26. It is known that the AER secretes fibroblast growth factor-4 (FGF4), which is essential for pattern specification of the limb skeleton ${ }^{[18]}$, and that the cells responsible for formation of the stylopodium, zygopodium and proximal autopodia are specified by stage $22^{[19]}$. The surface periderm cannot be a producer of FGF4, but its positive staining by PD antibodies is likely to be correlated with the functional activity of the AER. The morphology of the AER is also reported to follow this functional state ${ }^{[20,21]}$.

Anti-desmoplakin-1 staining performed in conjunction with PD immunohistochemistry revealed significant accumulation of desmoplakin-1 polypeptides in the periderm. This finding indicates that intercellular junctions mediated by desmosomes are more developed in the periderm than in the keratinizing cell layers at the stages examined, and suggests that the periderm may provide mechanical strength for the epidermis before sufficient development of desmosomes in the keratinizing cell layer. This assumption does not exclude reported possible functions assigned to the periderm recently ${ }^{[11-13]}$. Indeed, a large amount of glycogen in the periderm, suggesting active uptake from amniotic fluid, was also observed in the present study.

The immunoglobulin class of our antibodies, PD2, PD3, PD7 and PD9, was exclusively IgM, and these antibodies gave almost identical staining patterns as far as could be examined. Therefore, all of these antibodies could be used for detection of the chicken periderm immunohistochemically. For the human embryonic periderm, antibodies such as OC125, Peri-1 and GB1 have been generated and characterized as potential marker probes, although none of them are as specific to the periderm as our PD antibodies. CA125 antigen recognized by OC125 is present in the amnion, amniotic fluid and coelomic epithelium but not in the gut endoderm ${ }^{[7]}$. The Peri-1 epitope is detectable in apocrine glands, kidney tubules, thymus and endoderm-derived gut cells but not in the coelomic epithelium ${ }^{[22]}$. GB1 antibody raised against a simple extract of human amnion stains fetal esophagus and cornea but not bladder, oral mucosa or thymus ${ }^{[8]}$. Therefore, PD antibodies are useful tools for investigating the chicken periderm, although the antigen(s) recognized by our PDs could be different from those recognized by the human probes stated above. Future studies are needed to identify and characterize the PD antigen(s).

\section{Acknowledgements}

The authors gratefully acknowledge the technical and secretarial assistance of Mr. Hideo Nagai and Miss Yumi Kimura. Mouse myeloma (P3X63Ag8U.1; JCRB\#9085) used in the present study was obtained from the Japanese Cancer Research Resources Bank. This work was supported in part by a Grant-in-Aid from the Japanese Ministry of Education, Science and Culture (\#06671836) and Nihon University Research Grants (B94-100, B95-097, DA96-009). 


\section{References}

[ 1 ] Bickenbach, J. R., Greer, J. M., Bundman, D. S., Rothnagel, J. A. and Roop, D. R.: Loricrin expression is coordinated with other epidermal proteins and the appearance of lipid lamellar granules in development, J. Invest. Dermatol., 104, 405-410, 1995

[ 2 ] Pralomkarn, T., Nelson, J. and Gemmell, R. T.: Postnatal development of the skin of the marsupial native cat Dasyurus hallucatus, J. Morphol., 205, 233-242, 1990

[ 3 ] Holbrook, K. A. and Odland, G. F.: The fine structure of developing human epidermis: Light, scanning, and transmission electron microscopy of the periderm, J. Invest. Dermatol., 65, 16-38, 1975

[ 4 ] Watt, F. M., Keeble, S., Fisher, C., Hudson, D. L., Codd, J. and Salisbury, J. R.: Onset of expression of peanut lectin-binding glycoproteins is correlated with stratification of keratinocytes during human epidermal development in vivo and in vitro, J. Cell Sci., 94, 355-359, 1989

[ 5 ] Polakowska, R. R., Piacentini, M., Bartlett, R., Goldsmith, L. A. and Haake, A. R.: Apoptosis in human skin development: Morphogenesis, periderm, and stem cells, Dev. Dyn., 199, 176-188, 1994

[ 6 ] Vielkind, U., Sebzda, M. K., Gibson, I. R. and Hardy, M. H.: Dynamics of Merkel cell patterns in developing hair follicles in the dorsal skin of mice, demonstrated by a monoclonal antibody to mouse keratin 8, Acta Anat., 152, 93-109, 1995

[ 7 ] Nanbu, Y., Fujii, S., Konishi, I., Nonogaki, H. and Mori, T.: CA 125 in the epithelium closely related to the embryonic ectoderm: The periderm and the amnion, Am. J. Obstet. Gynecol., 161, 462-467, 1989

[ 8 ] Schofield, O. M., McDonald, J. N., Fredj-Reygrobellet, D., Hsi, B. L., Yeh, C. J., Ortonne, J. P. and Eady, R. A.: Common antigen expression between human periderm and other tissues identified by GB1-monoclonal antibody, Arch. Dermatol. Res., 282, 143-148, 1990

[ 9 ] Bickenbach, J. R. and Holbrook, K. A.: Proliferation of human embryonic and fetal epidermal cells in organ culture, Am. J. Anat., 177, 97-106, 1986

[10] Riddle, C. V.: Intramembranous response to cAMP in fetal epidermis, Cell Tissue Res., 241, $687-$ 689,1985

[11] Maruyama, T., Yoshizuka, M. and Fujimoto, S.: Light and electron microscopy of fetal rabbit skin with special reference to the role of mesenchymal cells in epidermal differentiation, Acta Anat., $133,143-155,1988$

[12] M'Boneko, V. and Merker, H.-J.: Development and morphology of the periderm of mouse embryos (days 9-12 of gestation), Acta Anat., 133, 325-336, 1988

[13] Scott, W. J., Jr, Nau, H., Wittfoht, W. and Merker, H. J.: Ventral duplication of the autopod: Chemical induction by methoxyacetic acid in rat embryos, Development, 99, 127-136, 1987

[14] Hamburger, V. and Hamilton, H. L.: A series of normal stages in the development of the chick embryo, J.Morphol., 38, 49-92, 1951

[15] Harlow, E. and Lane, D.: Antibodies: A Laboratory Manual, 1st ed., 139-244, Cold Spring Harbor Laboratory, New York, USA, 1988

[16] Giloh, H. and Sedat, J. W.: Fluorescence microscopy: reduced photobleaching of rhodamine and fluorescein protein complexes by $n$-propylgallate, Science, 217, 1252-1255, 1982

[17] Spurr, A. R.: A low-viscosity epoxy resin embedding medium for electron microscopy, J.Ultrastruct.Res., 26, 31-43, 1969

[18] Yang, Y. and Niswander, L.: Interaction between the signaling molecules WNT7a and SHH during vertebrate limb development: Dorsal signals regulate anteroposterior patterning, Cell, 80, 939-947, 1995

[19] Isokawa, K., Krug, E. L. and Markwald, R. R.: Leg bud mesoderm retains morphogenetic potential to express limb-like characteristics in collagen gel culture, Dev.Dyn., 193, 314-324, 1992 
[20] Todt, W. L. and Fallon, J. F.: Development of the apical ectodermal ridge in the chick leg bud and a comparison with the wing bud, Anat.Rec., 215, 288-304, 1986

[21] Todt, W. L. and Fallon, J. F.: Development of the apical ectodermal ridge in the chick wing bud, J.Embryol.Exp.Morph., 8, 21-41, 1984

[22] Lane, A. T., Negi, M. and Goldsmith, L. A.: Human periderm: A monoclonal antibody marker, Curr. Probl. Dermatol., 16, 83-99, 1987 